\title{
LIMITS ON COLD DARK MATTER CANDIDATES FROM AN ULTRALOW BACKGROUND GERMANIUM SPECTROMETER
}

\author{
S.P. AHLEN ${ }^{\text {a }}$, F.T. AVIGNONE III ${ }^{\text {b }}$, R.L. BRODZINSKI ${ }^{\text {c }}$, A.K. DRUKIER ${ }^{\mathrm{d}, \mathrm{e}}$, G. GELMINI ${ }^{\mathrm{f}, \mathrm{g}, 1}$ \\ and D.N. SPERGEL d,h \\ a Department of Physlcs, Boston University, Boston, MA 02215, USA \\ - Department of Physics, University of South Carolına, Columbia, SC 29208, USA \\ - Pactfic Northwest Laboratory, Richland, WA 99352, USA \\ d Harvard-Smithsonian Center for Astrophysics, Cambridge, MA 02138, USA \\ - Applied Research Corp., 8201 Corporate Dr, Landover MD 20785, USA \\ $\checkmark$ Department of Physics, Harvard University, Cambridge, MA 02138, USA \\ g The Enrico Fermi Institute, University of Chicago, Chicago, IL 60637, USA \\ h Institute for Advanced Study, Princeton, NJ 08540, USA
}

Received 5 May 1987

\begin{abstract}
An ultralow background spectrometer is used as a detector of cold dark matter candidates from the halo of our galaxy Using a realistic model for the galactıc halo, large regions of the mass-cross section space are excluded for important halo component partıcles. In particular, a halo domınated by heavy standard Dirac neutrınos (taken as an example of partıcles with spın-1ndependent $Z^{0}$ exchange interactions) with masses between $20 \mathrm{GeV}$ and $1 \mathrm{TeV}$ is excluded. The local density of heavy standard Dirac neutrinos is $<0.4 \mathrm{GeV} / \mathrm{cm}^{3}$ for masses between $17.5 \mathrm{GeV}$ and $25 \mathrm{TeV}$, at the $68 \%$ confidence level.
\end{abstract}

Galactic rotation curves suggest that most of the matter in the universe is non-luminous [1]. A variety of arguments suggest that this matter may be nonbaryonic [2]. This letter discusses the use of an ultralow background germanium spectrometer as a detector of cold dark matter particles interacting with Ge nuclei. Since only ${ }^{73} \mathrm{Ge}$, with a natural abundance of $7.8 \%$, has a non-zero spin, our best bounds apply to spin-independent (s.i.) interactions. Bounds on dark matter candidates coupling to baryons through $\mathrm{Z}^{0}$ exchange, like stable massive Dirac neutrinos [3] and scalar neutrinos [4], are presented. Our results exclude a halo dominated by particles with scattering cross section $\sigma^{\mathrm{s}}=\sigma_{\text {weak }}$ with masses $20 \mathrm{GeV}$ $\leqslant m \leqslant 1 \mathrm{TeV}$ (their local density is $<0.4 \mathrm{GeV} / \mathrm{cm}^{3}$ for $17.5 \mathrm{GeV} \leqslant m \leqslant 2.5 \mathrm{TeV}$ at the $68 \%$ confidence level) and apply to s.i. reactions in the range of $\sigma^{\mathrm{s} 1} \simeq 10^{-1} \sigma_{\text {weak }}$ to $\sigma^{\mathrm{s} 1} \approx 10^{-28} \mathrm{~cm}^{2}$ (for which the dark matter particles would be stopped in the earth's

\footnotetext{
1 On leave of absence from Department of Physics, University of Rome II, Via Orazio Raimondo, I-00173 Rome, Italy
}

crust before arriving at the detector) where $\sigma_{\text {weak }}$ is the weak scattering cross section of a standard heavy Dirac neutrino from a $\mathrm{Ge}$ nucleus. This range includes neutral technibaryons, recently proposed as dark matter candidates [5], having cross sections $\simeq 10 \sigma_{\text {weak }}$, which are, therefore, excluded for masses larger than $16 \mathrm{GeV}$. The ${ }^{73} \mathrm{Ge}$ in the detector with $s=9 / 2$, allows us to obtain a bound on particles with spin-dependent (s.d.) interactions, which case applies to particles in the range $\sigma \simeq 10^{4} \sigma_{\text {weak }}^{\text {s, }}$ to $\sigma \approx 10^{-28} \mathrm{~cm}^{2}$ (where $\sigma_{\text {weak }}^{\text {sd. }}$ corresponds to a standard heavy Majorana neutrino).

The measurement of the nuclear recoil, due to the scattering of heavy weakly interacting massive particles (WIMPs), requires a detector with a low energy threshold and excellent background rejection [6-8]. In this paper, the use of a germanium diode detector to search for dark matter is discussed. The low band gap $(0.69 \mathrm{eV}$ at $77 \mathrm{~K})$ and high efficiency for converting electronic energy loss to electron-hole $(\mathrm{e}-\mathrm{h})$ pairs $(2.96 \mathrm{eV}$ per e-h pair at $77 \mathrm{~K})$ make germanium detectors probably the best existing detectors 
of low velocity recoiling nuclei. Their low intrinsic noise $(\approx 500 \mathrm{eV}$ for equivalent electron energies of order $10 \mathrm{keV}$ ) make them ideal for the search for the rare phenomena considered in this paper as well as other exotica such as neutrinoless double beta decay.

An important parameter for the interpretation of count rates of recoiling nuclei in Ge detectors is the relative efficiency factor (R.E.F). This is the ratio of the number of $\mathrm{e}-\mathrm{h}$ pairs produced by an incident electron with energy, $T$, to the number of e-h pairs produced by an incident Ge nucleus with the same energy. The R.E.F. is equal to 1 only for $T \gg 1 \mathrm{MeV}$. For example, a uranium nucleus with energy $6 \mathrm{MeV}$, incident on a silicon diode, produces a signal equivalent to a $2 \mathrm{MeV}$ electron. This "pulse height defect" is attributed to (i) the energy loss of the ion in the electrode or detector dead layer; (ii) electron-hole recombination in regions of intense energy loss and/or poor collection of electrons; and (iii) com- petition between electronic and nuclear stopping powers. The first effect is not important for Ge recoils produced internally. Since the electronic energy loss 1s small for low velocities, the second effect is also not significant. Effect (iii) will therefore dominate in our case.

The R.E.F. for Ge detectors was calculated by evaluating the fraction of primary Ge recoil energy lost in electronic collisions. The electronic energy loss of secondary and higher order recoil nuclei was accounted for, and current electronic and nuclear stopping cross sections [9] have been used. Results are shown in fig. 1 (as function of the Ge nuclei energy recoil). The solid curve is based on the assumption that electronic energy loss is proportional to velocity at arbitrarily small velocities (1.e. the band gap is 1gnored), while the dashed curve is the result for a kinetic threshold energy of $0.27 \mathrm{keV}$ (a Ge nucleus with this energy transfers a maximum

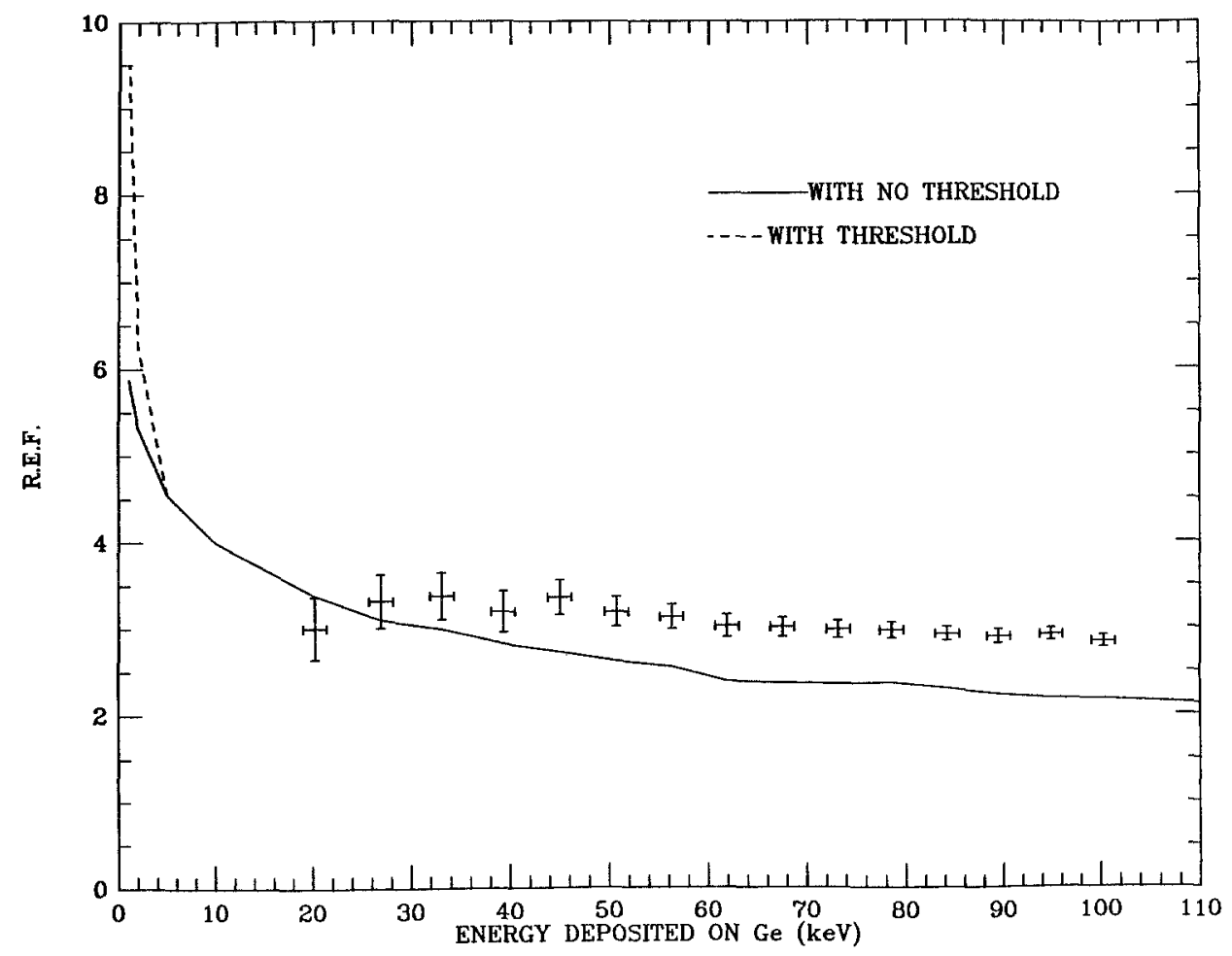

Fig. 1. The R.E.F. (the ratio of the number of electron hole-pairs produced by an incident electron to the number produced by a recoiling Ge nucleus with the same energy) is shown as function of Ge recoll energy. The solid curve is based on the assumption that there is no kinematic threshold, while the dashed curve shows the results for a kinematic recoll energy threshold of $0.27 \mathrm{keV}$ The theoretical curve is compared to data from Chasman et al. [10]. 
energy of $0.7 \mathrm{eV}$ to an electron in a direct collision, i.e. the band gap is regarded as a sharp threshold). In this way the sensitivity of the calculations to threshold phenomena is tested. The theoretical values were compared to data from Chasman et al. [10] who measured $\mathrm{e}-\mathrm{h}$ yields of neutron induced recoil nuclei within a Ge detector (fig. 2). Good agreement between the calculation and the data gives us confidence that the theoretical predictions should be valid down to several $\mathrm{keV}$. A similar experiment, on silicon instead of Ge, by Sattler [11], and other calculations of the expected response in $\mathrm{Ge}$ and $\mathrm{Si}$, by Linhard et al. [12], support our results. Linhard's calculations, which do not include higher than second-order nuclear collisions and use older data on cross sections, give lower values for the R.E.F. of $\mathrm{Ge}$ than the present calculation, by at most $12 \%$ for nuclear recoil energies between 20 and $100 \mathrm{keV}$.

The PNL/USC group has developed a $135 \mathrm{~cm}^{3}$ intrinsic Ge detector $[13,14]$ having a background reduced by about three orders of magnitude over conventional low background gamma-ray spectrometers. The detector is located in the Homestake mine at a depth equivalent to $4000 \mathrm{~m}$ of water to eliminate the cosmic ray induced background. The detector cryostat is constructed from high-purity copper and is surrounded by 11 tons of lead, sheet cadmium and neutron moderator, to eliminate the radioactive background and neutrons from the rock. The inner shield was made from high purity copper, when the $14 \mathrm{~d}$ of data used in this work were taken. These data were selected because they correspond to a period of decreased level of mining operations in the vicinity of the detector. This resulted in fewer microphonic noise pulses. For this analysis, the absence of low energy noise is more important than the quantity of data. However, $1000 \mathrm{~h}$ of data are shown in fig. 2 to better display the $\mathrm{X}$-ray and $\gamma$-ray peaks used for energy calibration.

The energy threshold was reduced to an incident electron energy of $4 \mathrm{keV}$. According to fig. 1, this permitted the detection of Ge nuclei-WIMP scatterings with nuclear recoil energies greater than 15 $\mathrm{keV}$. The count rates of $1000 \mathrm{~h}$ of low energy data are shown in fig. 2, as function of incident electron energy ( to be multiplied by the appropriate R.E.F to get the corresponding nuclear recoil energy). The following photon peaks, clearly in evidence, were

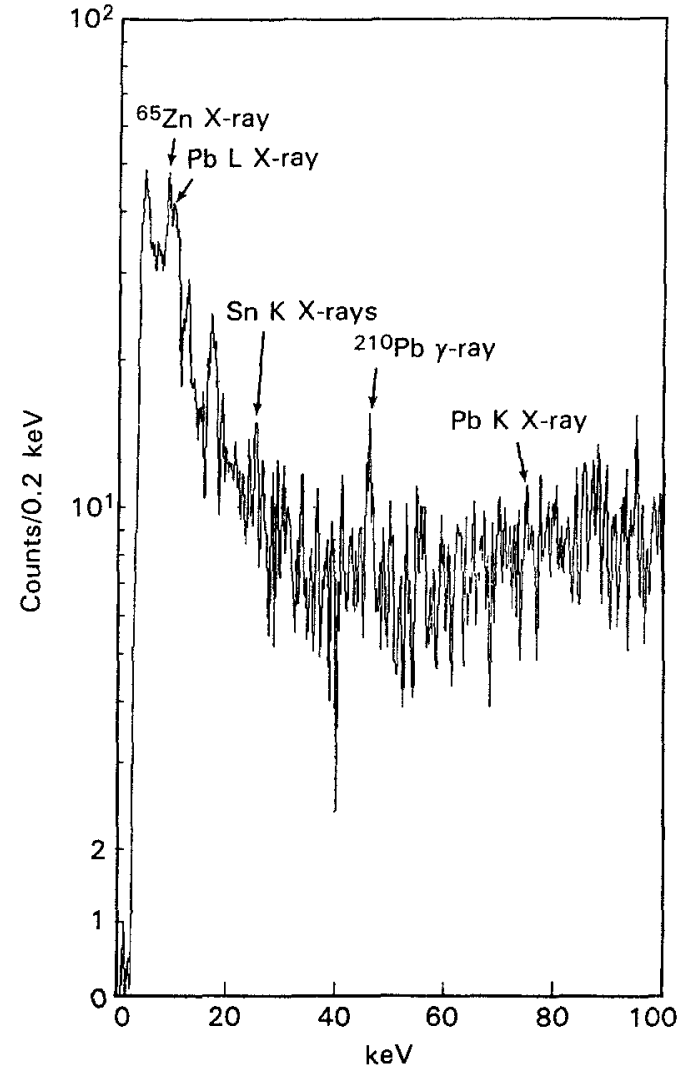

Fig. 2. $1000 \mathrm{~h}$ of data from the $\mathrm{Ge}$ spectrometer are shown The width of each channel is $0.2 \mathrm{keV}$. The identıfied peaks result from the decay products of radioactivity in the exposed solder.

used to calibrate the detector: the $8.9805 \mathrm{keV} \mathrm{Cu} \mathrm{X-}$ ray from the electron capture of ${ }^{65} \mathrm{Zn}$, the $10.551 \mathrm{keV}$ $\mathrm{Ga} X$-ray from the electron capture of ${ }^{68} \mathrm{Ge}$ plus $\mathrm{Pb}$ $\mathrm{L}$, the $25.2713 \mathrm{keV}$ Sn K X-ray, the $46.503 \mathrm{keV} \gamma$ ray in ${ }^{210} \mathrm{Bi}$ from the decay of ${ }^{210} \mathrm{~Pb}$ and the 74.969 $\mathrm{keV} \mathrm{Pb}$ X-ray. The $\mathrm{Pb}$ and $\mathrm{Sn} \mathrm{X}$-rays came from a solder connection in the proximity of the detector. $\mathrm{The} \mathrm{Cu}$ and $\mathrm{Ga} \mathrm{X}$-rays are sums of $\mathrm{X}$-rays from all shells, since the sources were cosmogenically created within the crystal. Each line was fitted with a modified gaussian to obtain its centroid. Then the ratio of the channel to energy of the peak was fitted to a quadratic function. There is a $0.1 \%$ non-linearity. The spectrum was then adjusted until the best fit was achieved. The non-linearity accounts for about one channel in one thousand. The maximum error in the calibration at $4 \mathrm{keV}$ is one half of a channel, which is $0.1 \mathrm{keV}$. 
The observed count rate can be compared to the rate predicted if the halo was comprised of WIMPs. The model of the halo used assumes a local halo density of 0.01 solar mass $/ \mathrm{pc}^{3}=0.38 \mathrm{GeV} / \mathrm{cm}^{3}[15]$, and an isotropic (in the halo rest frame) gaussian distribution function of velocities of the halo particles, $f(v)$, with an RMS of $250 \mathrm{~km} / \mathrm{s}$ and a maximum of $550 \mathrm{~km} / \mathrm{s}$ [8]. It is assumed that the halo, slowly rotates with a local velocity of $70 \mathrm{~km} / \mathrm{s}$ [16], like the galactic spheroid. This is a conservative assumption which reduces relative velocities since the Sun moves around the galaxy in the same sense, at $250 \mathrm{~km} / \mathrm{s}$; the halo may not rotate at all. The maximum halo velocity may be higher [15] in which case the limits extend to lower masses. The local halo density is known with a factor of 2 uncertainty. The predicted rate of recoils having energy $T$ and producing a signal in the detector, $R_{\mathrm{p}}(T)$, was calculated according to

$$
R_{\mathrm{p}}(T)=n_{\mathrm{X}} \Delta T \int_{v_{\min }(\mathrm{T})}^{\nu_{\max }} \frac{\mathrm{d} \sigma}{\mathrm{d} T}(v, T) f(\boldsymbol{v}) v \mathrm{~d}^{3} v,
$$

where $\Delta T$ is the range of recoil energies detected in a given channel centered in $T$ and $n_{\mathrm{X}}$ is the local density of dark matter. Standard Dirac neutrinos were chosen as an example of coherently interacting particles. The cross section as a function of recoil energy, $\mathrm{d} \sigma / \mathrm{d} T$, for an incident standard Dirac neutrino (that interacts through $Z^{0}$ exchange) depends upon its mass, $m_{\mathrm{x}}$, and velocity, $v$, according to

$$
\begin{aligned}
\frac{\mathrm{d} \sigma}{\mathrm{d} T} \simeq & \simeq \frac{G_{\mathrm{F}}^{2} m_{\mathrm{N}} c^{2}}{8 \pi v^{2}}\left[Z\left(1-4 \sin ^{2} \theta_{\mathrm{W}}\right)-N\right]^{2} \\
& \times\left[1+\left(1-\frac{T}{E_{\mathrm{x}}}\right)^{2}-\frac{m_{\mathrm{N}} T+m_{\mathrm{x}}^{2}}{E_{\mathrm{x}}^{2}}\right] \\
& \times \exp \left(-m_{\mathrm{N}} 2 T R^{2} / 3 \hbar^{2}\right),
\end{aligned}
$$

where $m_{\mathrm{N}}$ is the mass of the nucleus, $Z$ and $N$ are the atomic and neutron numbers respectively, $T$ is the recoil energy, $G_{\mathrm{F}}$ is the weak coupling constant, $\theta_{\mathrm{W}}$ is the weak mixing angle and $E_{\mathrm{x}}=m_{\mathrm{x}}\left(c^{2}+v^{2} / 2\right)$. When the de Broglie wavelength $1 / q$ of the momentum transfered in the recoil $g=\left(2 m_{\mathrm{N}} T\right)^{1 / 2}$, is smaller than the nucleus, the assumption of a coherent interaction with a point-like nucleus is no longer valid, and the finite size of the nucleus must be included
[17]. The exponential in eq. (2) is a nuclear form factor corresponding to a gaussian density distribution of nucleons, in a nucleus of radius $R=1.2 A^{1 / 3}$ $\mathrm{fm}$, and atomic number $A$ [17]. A better model of the nucleonic density, such as a Woods-Saxon density [18], would produce a form factor decreasing less steeply with $T$. Note that for small energy transfers, $T \ll 11 \mathrm{keV}$, such that $q R \ll 1$, the WIMP interacts with a point-like nucleus, and there is no loss of coherence. The halo model used is conservative. If the maximum halo velocity was closer to the local escape velocity of $\sim 750 \mathrm{~km} / \mathrm{s}$ [15], the lower limit of the excluded mass range would be $10 \mathrm{GeV}$.

The observed count rate in the germanium detector can be used to obtain limits on the density of interacting dark matter particles in the halo. The integral in eq. (1) was evaluated over all velocity phase space and bounds on $n_{\mathrm{X}}$, at the $68 \%$ and $95 \%$ confidence levels, were obtained for every $T$. The most restrictive $T$ dependent bounds were taken as the final bounds on $n_{\mathrm{X}}$. The best bounds come from values of $T$ near threshold, because of the rapid decrease of the predicted rate with increasing $T$ (notice that in the non-relativistic limit $(\mathrm{d} \sigma / \mathrm{d} T)^{\mathrm{NR}}=\sigma^{\mathrm{NR}} / T_{\max }$, where $\sigma^{\mathrm{NR}}$ does not depend on $T$, and for a given $T$ one must sum over all $T_{\max }>T$ ). Because of their importance in this analysis, the number of counts in the first ten channels (of $0.2 \mathrm{keV}$ each), of the $14 \mathrm{~d}$ of data used, starting at $4.0 \mathrm{keV}$ are given. They were respectively 4.53 , $9.65,19.38,20.66,15.78,16.33,16.84,13.46,12.01$, 10.64. Fig. 3 shows the limits for standard heavy Dirac neutrinos, which have s.i. $Z^{0}$ exchange interactions. Fig. 3 can be used for other s.i. vectorial interactions by multiplying the vertical axis by the ratio $\left(\sigma_{\text {weak }} / \sigma^{s 1}\right)$. For example for neutral technibaryons this ratio would be approximately 0.1 and for sneutrinos this ratio is 0.5 . Similar curves for s.d. interactions will be given in a subsequent paper. The spectrum has a smooth continuum contribution, due mainly to Compton-scattered background $\gamma$-rays. The low energy peaks in the present data are primarily due to the presence of the ${ }^{210} \mathrm{~Pb}$ decay chain, in a solder connection in direct line-of-sight to the surface of the detector. The solder and an indium contact have recently been removed, and the inner shield has been upgraded by the use of $449 \mathrm{y}$ old lead in the place of the super-pure copper, which had some 


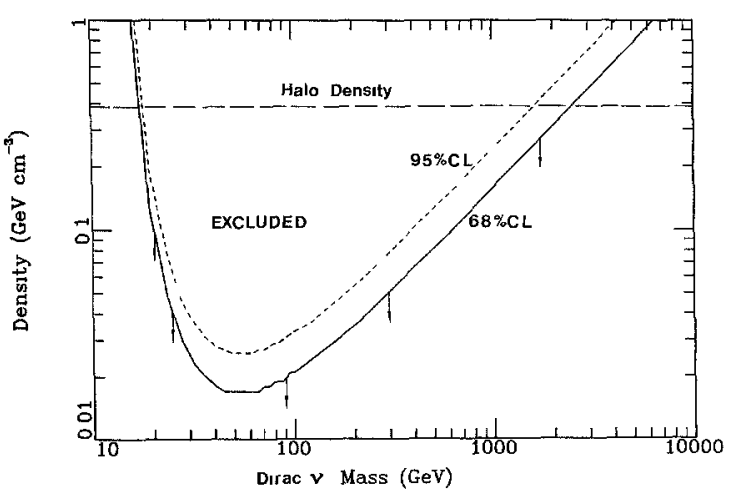

Fig. 3. The maximum halo density of heavy standard Dirac neutrinos (as an example of particles with weak spin-independent interactions) is shown, consistent with the observed count rate, as a function of their mass. The solid line shows the $68 \%$ confidence level and the dashed line shows the $95 \%$ confidence level.

cosmogenic radioactive contamination. In this way the background has been reduced by about a factor of ten at around $50 \mathrm{keV}$. The reduction of background appears to improve with decreasing energy; however, in an attempt to reduce background from ${ }^{40} \mathrm{~K}$, data are being acquired in the present phase with the field-effect transistor removed from the cryostat. This configuration increases the low energy noise (while not reducing the ${ }^{40} \mathrm{~K}$ significantly). New data on dark matter will not be available untill the next phase of the experiment.

When the data for this work were taken, the energy threshold was set at $4 \mathrm{keV}$ because of microphonic noise at lower energies. The shape of the low energy $\mathrm{X}$-ray lines suggests that $\Delta E(\mathrm{FWHM}) \approx 500 \mathrm{eV}$ in this region. The strong increase of noise below $E_{\mathrm{th}}=4$ $\mathrm{keV}$ is largely due to microphonics engendered by mining operations. Hardware and software have recently been developed to reduce this noise and permit lowering the energy threshold to $1 \mathrm{keV}$. In the near future, rejection/detection of the existence of coherently interacting particles of mass $>8 \mathrm{GeV}$ should be feasible. Detection could be confirmed by the expected modulation in the signal due to the earth's motion relative to the halo [8].

It will be difficult to reduce the energy threshold below $1 \mathrm{keV}$, thus the detection of particles of lower mass will require cryogenic detectors. The germanium detector is also not sensitive to particles like the photino that couple through s.d. weak interactions.

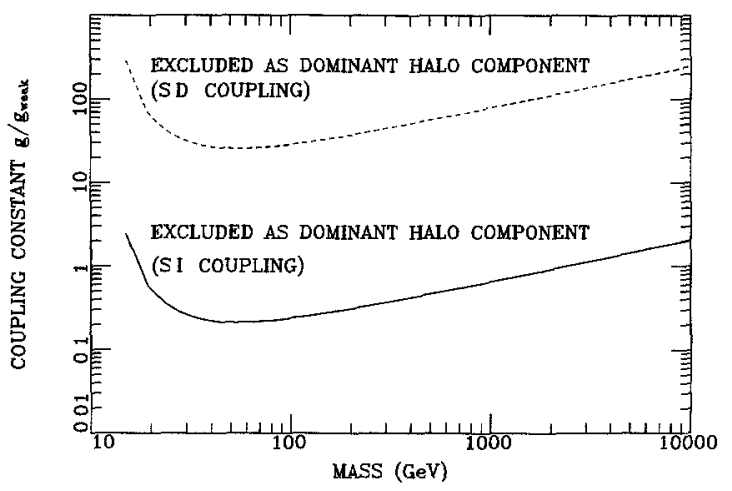

F1g. 4. The regions in mass-cross section space excluded at the $68 \%$ confidence level are shown. The halo cannot be composed of particles that interact with nuclei through spin-independent interactions whose coupling constant (normalized to the coupling of massive Dirac neutrinos to baryons) lies above the solid line. Nor can the halo be composed of particles that interact with nuclel through spin-dependent interactions whose coupling constant (normalized as above) lies above the dashed line.

Our main results are shown in fig. 4 . The range of mass and cross section of particles excluded as main components of the halo are shown. The ratio $g / g_{\mathrm{w}}$ is defined as $\left(\sigma / \sigma_{\text {weak }}\right)^{1 / 2}$ where $\sigma_{\text {weak }}$ is the cross section for standard heavy Dirac neutrinos.

A limit on the solar axion flux has also been derived from the USC/PNL germanium spectrometer [19].

F.T.A. is supported by NSF grant PHY-8405654, R.L.B. by DOE Contract DE-AC06-76RLO-1830, G.G. by NSF grants PHY-82-15249 and PHY-8521588 and DOE grant DE-AC02-82ER-40073, and DNS by NSF grants PHY-83-06693 and PHY-8217352. We would like to thank R. Glauber and T. Jaroszewicz for useful discussions.

\section{References}

[1] S M. Faber and J.S Gallagher, Annu. Rev. Astron Astrophys 17 (1979) 135,

D. Burnstein and V.C. Rubin, Astrophys. J. 297 (1985) 423.

[2] D.J. Hegy1 and K.A. Olive, Phys. Lett. B 126 (1983) 28; Astrophys J. 303 (1986) 56.

[3] J. Bagger, S Dimopoulos, E Masso and J. Reno, Phys. Rev. Lett. 54 (1985) 2199;

E. Kolb and K. Olive, Phys. Rev. D 33 (1986) 1202; D 34 (1986) 2531.

[4] J.S. Hagelın, G.L. Kane and S. Raby, Nucl. Phys. B 241 (1984) 648, L E Ibáñez, Phys. Lett B 137 (1984) 160.

[5] S Nussinov, Phys. Lett. B 165 (1985) 55 
[6] M.W. Goodman and E. Witten, Phys. Rev. D 31 (1985) 3059; I Wasserman, Phys Rev. D 33 (1986) 2071.

[7] A.K. Drukıer and L. Stodolsky, Phys. Rev D 30 (1984) 2295.

[8] A.K. Drukier, K. Freese and D.N. Spergel, Phys. Rev. D 33 (1986) 3495

[9] W.D. Wilson, L.G. Haggmark and J.P. Biersack, Phys. Rev. B 15 (1977) 2485.

[10] C. Chasman, K.W. Jones and R.A. Ristınen, Phys. Rev. Lett. 15 (1965) 245.

[11] R.A. Sattler, Phys Rev. 138 (1965) A1815.

[12] J. Linhard, V. Nielsen, M. Scharff and P.V. Thompsen, Kgl. Danske Videnskab Selskab Mat. Fys. Medd 33, No. 10 (1963).

[13] R.L. Brodzınskı, D.P. Brown, J.C. Evans Jr,, W.K Hensley, J.H. Reeves, N.A Wogman, F T. Avignone III and H.S. M1ley, Nucl. Instrum. Methods A 239 (1985) 207.
[14] F.T. Avıgnone III, R.L. Brodzınskı, J.C. Evans Jr., W.K. Hensley, H.S. Miley and J.H. Reeves, Phys. Rev. C 34 (1986) 666

[15] J. Caldwell and J.P. Ostriker, Astrophys. J. 251 (1981) 61. [16] J. Bahcall and S. Casertano, Astrophys. J. 308 (1986) 347.

[17] D.Z. Freedman, Phys. Rev. D 9 (1974) 1389; D. Tubbs and D.N. Schramm, Astrophys. J. 201 (1975) 467.

[18] E.g. R C. Barrett and D.F. Jackson, Nuclear sizes and structure (Clarendon, Oxford, 1979).

[19] F.T. Avıgnone III, R.L. Brodzinski, S. Dimopoulos, A.K. Drukier, G. Gelmını, B.W. Lynn, D.N. Spergel and G.D. Starkman, Limits on solar axions from the ultralow background germanium spectrometer, SLAC-Pub. 3872 and PNL-SA-14132(1986), Phys. Rev. D, to be published. 